DOI: $10.21767 / 2573-5365.100014$

\title{
Molecular Imaging as a Complementary Tool for Personalized Molecular Medicine: Inception to Proven Proactive Approach for Better Treatment
}

\author{
Amitabha Acharya ${ }^{* 1,2}$ \\ ${ }^{1}$ Biotechnology Division, CSIR-Institute of Himalayan Bioresource Technology, Palampur (H.P.) 176061, India \\ ${ }^{2}$ Academy of Scientific and Innovative Research (AcSIR), New Delhi, India \\ ${ }^{*}$ Corresponding author: Amitabha Acharya, Biotechnology Division, CSIR-Institute of Himalayan Bioresource Technology, Palampur (H.P.) \\ 176061, India, Tel: +91-1894-233339; E-mail: amitabhachem@gmail.com
}

Rec date: April 25, 2016; Acc date: April 27, 2016; Pub date: April 29, 2016

Citation: Acharya A. Molecular Imaging as a Complementary Tool for Personalized Molecular Medicine: Inception to Proven Proactive Approach for Better Treatment. Cell Mol Med. 2016, 2:1.

\section{Editorial}

Personalized medicine refers to the tailoring of medical treatment to the individual characteristics, needs and preferences of each patient. The concept of such treatment strategies have originated from the observations of the clinicians that patients with identical symptoms may have different illnesses, with different causes and consequently, the medical interventions requires special attention for each individual.

Over a period of time this particular field has seen merger of a wide range of fields ranging from genomics to medical imaging to regenerative medicine etc. Molecular medicine which emphasizes the cellular and molecular phenomena and interventions of the disease to individualize treatment and improve patient care may easily be correlated with personalized medicine. In the near future personalized molecular medicine will ensure that the patients receive the right medication, in the right amount for the right disease.
The successful implementation of this new treatment strategy is paving the path for developing new areas in fields of diagnostic medicine viz., imaging and pathology. Both imaging and pathology of the disease site contribute significantly in monitoring disease progression. Molecular imaging is a research discipline that visualizes, characterizes, and quantifies the biological processes at the cellular and sub-cellular domain at physiological environment and allows early diagnosis and monitors curative effects.

Molecular imaging takes the help of biomarkers or other objective indicators of pathogenic processes to forecast or monitor healing responses. The objective of molecular imaging suggests significant overlap with the interest of molecular pathology and thus, it can act as a complementary tool for molecular medicine. Researchers around the world foresee that the next decade will observe unprecedented development in the molecular imaging techniques, which in succession, is expected to have huge impact on personalized molecular medicine. 